\title{
The Pharmacokinetics and Skin Changes in Rabbit on Selamectin Solution
}

\author{
Yu Chuan ${ }^{1, ~ *, ~ L i u ~ P e n g g a n g ~}{ }^{2}$, Yang Gang ${ }^{1}$, Wu Qiang ${ }^{1}$ \\ ${ }^{1}$ College of Agricultural, Yibin Vocation \& Technical College, Yibin, China \\ ${ }^{2}$ College of Veterinary Medicine, Yang Zhou University, Yangzhou, China \\ Email address: \\ 1254021273@qq.com (Yu Chuan), 505392665@qq.com (Liu Penggang) \\ ${ }^{*}$ Corresponding author
}

\section{To cite this article:}

Yu Chuan, Liu Penggang, Yang Gang, Wu Qiang. The Pharmacokinetics and Skin Changes in Rabbit on Selamectin Solution. International Journal of Animal Science and Technology. Vol. 5, No. 2, 2021, pp. 32-37. doi: 10.11648/j.ijast.20210502.11

Received: March 23, 2021; Accepted: April 10, 2021; Published: April 20, 2021

\begin{abstract}
Background: It found that many antiparasitic drugs have a good treatment effect on the pets skin diseases. whether selamectin solutions have a bad effect on skin tissues needs more research. Objective: In order to investigate the effect of selamectin solution on skin changes in rabbit. Method: the skin of rabbit was given different doses of selamectin solution, then the quantity of per unit area of hair follicles and sebaceous glands and the morphology structure of them and the changes of skin thickness, epidermis thickness and dermis thickness were researched through routine paraffin sections and H. E, Evg, Masson's, Pas, Toluidine blue staining and Image Pro Plus6.0. The concentration of blood and urine were measured by High Performance Liquid Chromatography after administration. Results: The thickness of skin, per unit of hair follicles and sebaceous glands all had an obviously increasing trend with the increasing of doses. 1 day later, the blood and urine concentration of the selamectin and the metabolite avermectin all were in highest. Many selamectin solution were excluded in the form of prototypes and many were in avermectin. There were a higher blood concentration and metabolite avermectin until 72h. Conclusion: The skin appearance changes were insignificant while the skin thickness and accessory organs increased obviously in microscope after given more selamectin. In $24 \mathrm{~h}$, the blood and urine concentration all were in highest. This study, which discussed the influences by selamectin solution in rabbit skin and the pharmacokinetics, was to provide the basis for the deeper study of Antiparasitic drugs effects on pet skin diseases.
\end{abstract}

Keywords: Rabbit, Epidermis, Dermis, Hair Foallicles, Sebaceous Glands, Pharmacokinetics

\section{Introduction}

Selamectin which belongs to macrolides antibiotics is a good insecticide in pet parasites inside and outside. M. Honda el have made a research that selamectin solution was a suitable and economical treatment for guinea pigs infected with the mites in doses of $5.0 \sim 7.5 \mathrm{mg} /$ head administration [1]. James W el found selamectin solution can be absorbed transdermally through skin and be immediately eliminated in rabbits while the long-term safety and repeated applications must be certified [2]. Jennifer J. D'Agostino el foud selamectin can be absorbed obviously in bullfrogs skin, single doses administration had no significant histologic effects even if in high concentrations [3]. Sang-Hun Kim founed at a dose of $12 \mathrm{mg} / \mathrm{kg}-1$ is effective and convenient for controlling cheyletiellosis in rabbits and may be a practical and well-tolerated treatmentoption in this species [4]. And Arif Kurtdede's study was similar in rabbits in P. cuniculi and S. scabiei [5]. Selamectin also show a good effect on mite infection in masked palm civet and in additional endo- and ectoparasites especially in heartworm disease in cats $[6,7]$. In all, Selamectin can exert an ideal deworming effects through the skin administration.

The skin in rabbit consists of epidermis, dermis and subcutaneous tissue, hair follicles, sebaceous glands, sweat glands, etc. The skin are often sufferred from infectious diseases, such as bacterium which contains Staphylococcus Necrobacillosis, fungus like dermatophytosis and microsporum canis [8-11]. Temporary parasites contains Sarcoptidae, Psoroptidae, flea etc which can affect the rest and feeding then causing lowly development and no 
economic value [12]. Up to now, there is no report on the effect of selamectin solution on rabbit skin tissue structure. This study was to measure the skin thickness of the site in administration and the opposite, to research the changes in hair follicles and sebaceous glands and to measure the blood and urine concentration and metabolite. It could provide a reference for transdermal administration in pets, which summarize the effect of selamectin solution in rabbit skin.

\section{Materials and Methods}

\subsection{Sample Collection}

There were four doses administration groups in this study respectively $6 \mathrm{mg} / \mathrm{kg}, 18 \mathrm{mg} / \mathrm{kg}, 60 \mathrm{mg} / \mathrm{kg}$ and $120 \mathrm{mg} / \mathrm{kg}$. Rabbits and Drugs were bought in Yi Bin Xiehe Yijia animal hospital. Plasma was obtained by centrifuging blood and blood was gathered through heart after administration in $120 \mathrm{mg} / \mathrm{kg}$ group in $1 \mathrm{~h}, 2 \mathrm{~h}, 6 \mathrm{~h}, 12 \mathrm{~h}, 24 \mathrm{~h}, 48 \mathrm{~h}, 72 \mathrm{~h}$. The urines were collected by diapers in $2 \mathrm{~h}, 6 \mathrm{~h}, 12 \mathrm{~h}, 24 \mathrm{~h}, 48 \mathrm{~h}, 72 \mathrm{~h}$. In $72 \mathrm{~h}$, the rabbits were put to death, then taking skin samples immediately in longissimus site and the opposite.

\subsection{Reagents and Instruments}

Ethanol 99.99\%, Ethanol 95\%, dimethylbenzene 99.99\%, Paraformaldehyde $99.99 \%$, all bought from KeLong Huaxuepin company in Chendu. Hematoxylin and alcohol soluble were bought in Thermo Fisher company. Neutral environmentally friendly quick-drying glue was bought in Yulu Shiyanqicai company in Nanchang. Paraffin slicer, Thermostat water bath cauldron, light microscope, Leica microscopic imaging system, Anticoagulant tube, centrifugal machine, $-80^{\circ} \mathrm{C}$ refrigerator, High-performance liquid chromatography agilent.

\subsection{Samples Preparation}

Skin samples were fixed in 4\% paraformaldehyde solution, then washed by running water $30 \mathrm{~min}$ and embedded in paraffin, later making the $5 \mu \mathrm{m}$ thickness continue slices and staining. Plasma samples were collected by heart blood, then stored in $-80^{\circ} \mathrm{C}$ refrigerator after centrifugating. Urine was gathered by diapers, then dissolved in acetonitrile and also stored in $-80^{\circ} \mathrm{C}$ refrigerator.

\subsection{Data Measurement and Statistics}

Per unit of hair follicles and sebaceous glands were calculated and the skin thickness, epidermis thickness and dermis thickness were measured and the average value were counted by Image Pro Plus 6.0 after observing the tissue samples through Microscopic imaging system. Skin average thickness increasing by doses=skin average thickness in high doses- skin average thickness in low doses. Amount of hair follicles and sebaceous glands increasing=amount of hair follicles and sebaceous glands in high doses-amount of hair follicles and sebaceous glands in low doses. The greater changes were more significant in differential values. Everytime samples concentrations were calculated by concentration of reference substance, Peak area of reference substance and Samples peak area all were ensured by the peak time through High Performance Liquid Chromatography.

\section{Results}

\subsection{Skin Thickness Changes in Administration in Rabbits}

With the increasing administration, the skin thickness, epidermis thickness, dermis thickness shows a significantly addition trend. The skin thickness ranged from $518 \mu \mathrm{m} \sim 1279$ $\mu \mathrm{m}, 964 \mu \mathrm{m}$ average. The epidermis thickness ranged from $9.249 \mu \mathrm{m} \sim 29.352 \mu \mathrm{m}, 16.892 \mu \mathrm{m}$ average, and the dermis were $501.6 \mu \mathrm{m} \sim 1202.7 \mu \mathrm{m}, 926.5 \mu \mathrm{m}$. The addition trend was more obvious because of increasing doses. The thickness increasing degree were mainly due to the dermis changes. (Figure 1).
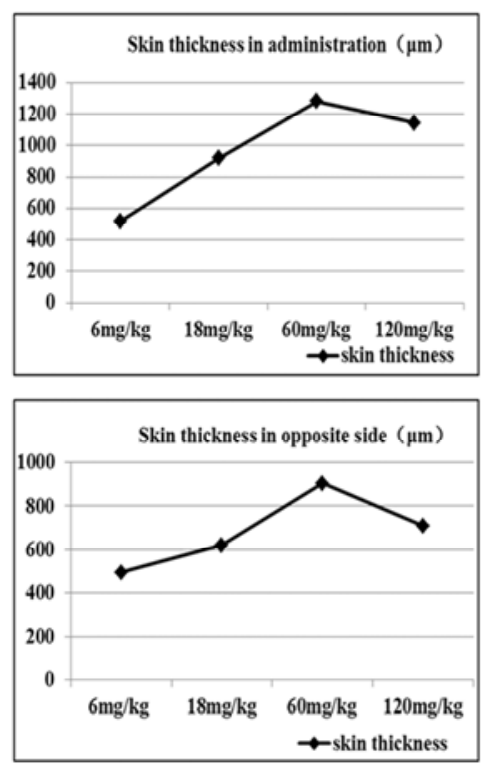
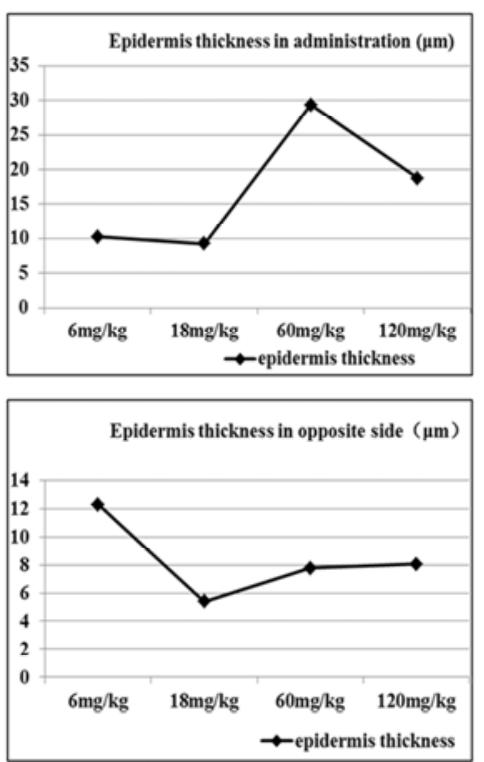
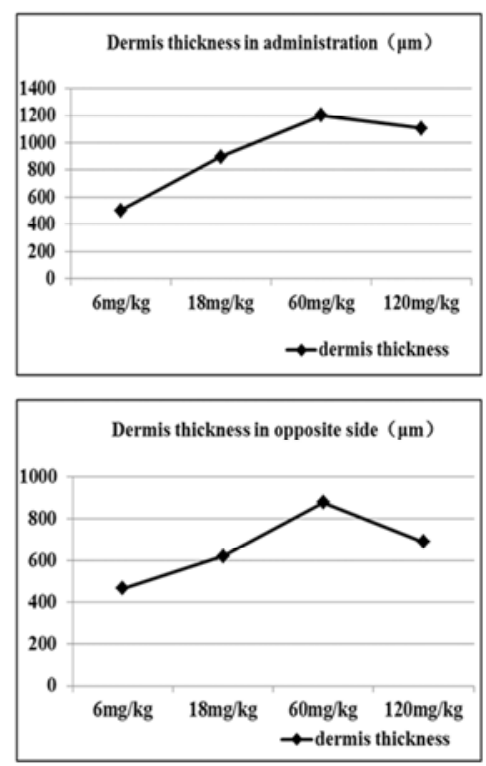

Figure 1. The thicknesses changes of skin in administration and opposite site in rabbit. 


\subsection{Skin Thickness Changes in Opposite Side in Rabbits}

The skin thickness, epidermis thickness, dermis thickness all showed an addition trend in the opposite side. The skin thickness ranged from $495.4 \mu \mathrm{m} \sim 903.83 \mu \mathrm{m}, 707.04 \mu \mathrm{m}$ average. The epidermis thickness ranged from 5.385 $\mu \mathrm{m} \sim 12.305 \mu \mathrm{m}, 8.380 \mu \mathrm{m}$ average, and the dermis were $467.235 \mu \mathrm{m} \sim 878.407 \mu \mathrm{m}, 664.111 \mu \mathrm{m}$. The addition trend was relatively insignificant and the thickness increasing degree was also mainly due to the dermis changes. (Figure 1)

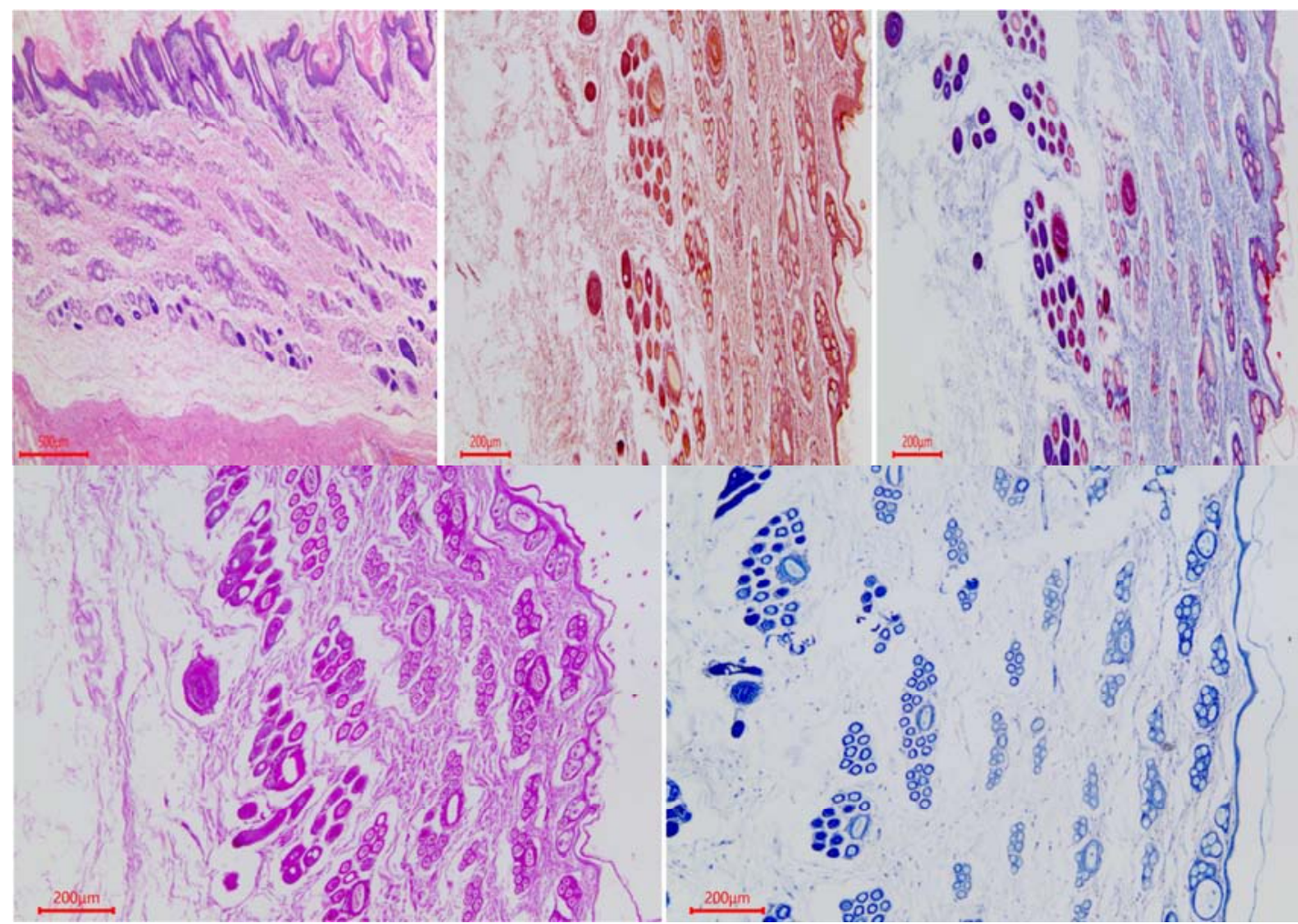

Figure 2. The staining of skin, containing H. E, EVG, Masson's, Pas, Toluidine bulue.

\subsection{Hair Follicles Changes}

The number of hair follicles showed a significantly addition trend in determined area with the increasing doses. Per unit of hair follicles ranged from $54.360 / \mathrm{mm} 2 \sim 140.441$ $/ \mathrm{mm} 2,105.358$ average, in $60 \mathrm{mg} / \mathrm{kg}$, the trend was espeacilly obvious. While the opposite sides were not apparently it ranged from $41.515 / \mathrm{mm}^{2} \sim 64.525 / \mathrm{mm}^{2}, 53.034$ average. The number of hair follicles in administration site increased significantly while the opposite were in normal. (Table 1)

\subsection{Sebaceous Glands Changes}

The number of sebaceous glands showed an apparently addition trend in determined area and the opposite side with the increasing doses. Per unit of sebaceous glands ranged from $0.406 / \mathrm{mm} 2 \sim 2.349 / \mathrm{mm} 2,1.428$ average, the opposite ranged from $0.365 / \mathrm{mm} 2 \sim 2.349 / \mathrm{mm} 2,1.232$ average. The number of sebaceous glands both in administration site and the opposite increased significantly. (Table 1)

Table 1. The number of hair follicles and sebacceous glands.

\begin{tabular}{lll}
\hline Group & Per unit of hair follicles $\left(/ \mathbf{m m}^{2}\right)$ & Per unit of sebaceous glands $\left(/ \mathbf{m m}^{2}\right)$ \\
\hline $6 \mathrm{mg} / \mathrm{kg}$ & 54.3598 & 0.406 \\
$6 \mathrm{mg} / \mathrm{kg}$ opposite & 64.525 & 0.365 \\
$18 \mathrm{mg} / \mathrm{kg}$ & 86.196 & 1.184 \\
$18 \mathrm{mg} / \mathrm{kg}$ opposite & 41.515 & 1.104 \\
$60 \mathrm{mg} / \mathrm{kg}$ & 110.432 & 1.789 \\
$60 \mathrm{mg} / \mathrm{kg}$ opposite & 56.860 & 1.670 \\
$120 \mathrm{mg} / \mathrm{kg}$ & 140.441 & 2.349 \\
$120 \mathrm{mg} / \mathrm{kg}$ opposite & 49.237 & 2.331 \\
\hline
\end{tabular}

\subsection{The Changes in Skin Thickness and Tissue Structure}

In all, the administration site skin shows a greatly increasing trend in skin thickness, dermis thickness, per unit of hair follicles and sebaceous glands through in high doses. Compared with the administration site, the opposite corresponding site trend was relatively weakened in these fields. The structure of skin tissues, hair follicles and 
sebaceous glands show no obvious changes through administration.

\subsection{Drug Concentration in Blood and Urine}

Selamectin was absorbed in blood through skin by skin administration and entered the whole body in blood circulation system. The selamectin and avermectin in blood were tested after administration, in $24 \mathrm{~h}$, both of them were in highest concentration, respectively $12.22 \mu \mathrm{g} / \mathrm{mL}$ and
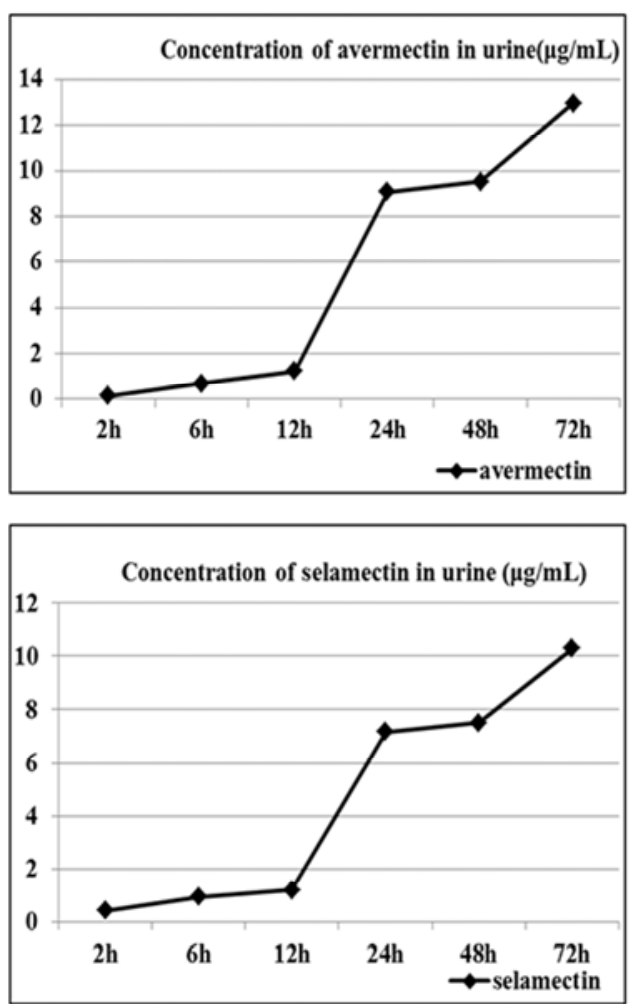

Figure 3. The concentration of selamectin and avermectin in blood and urine.

\section{Discussion}

This study suggests that regular concentration selamectin solution can cause clearly thickening in animal skin and the number of hair follicles and sebaceous glands obviously increasing, so the barrier function of the skin can be significantly enhanced. These results are the same with these drugs on skin function such as H. Hernandez's study on the function of pentyl-rhamnoside in skin's keratinocyte, J. Idkowiak 's roport on the starfruit (Averrhoa carambola) leaf extract and M. Hou's study on topical hesperidin which was from the Orange peel [13-15]. Some researches on many durgs were harmful to the skin barrier, like the Glucocorticoids, EGFR regulation and Histamine suppresses [16-18]. These activated substances had a special mechanism of action which were to repair the barrier function of the skin. The treatment on skin parasitic diseases is to cause rapid death of the worm to achieve the effects of broad spectrum. While in higher concentration and repeated dosing, especially in $80 \%$ and $100 \%$, which could cause the morphological changes, acute inflammatory and a large scale occurrence of
$9.64 \mu \mathrm{g} / \mathrm{mL}$. The selamectin and metabolite avermectin in urine which were collected and tested were in growing trend after administration in $2 \mathrm{~h}$. Selamectin solution can be absorbed in blood and distributed quickly in whole body which retains a high blood concentration within a certain time frame. Drugs were excreted many in prototype selamectin and many in metabolite avermectin through urine. The concentration in blood and urine were in figure 3 .
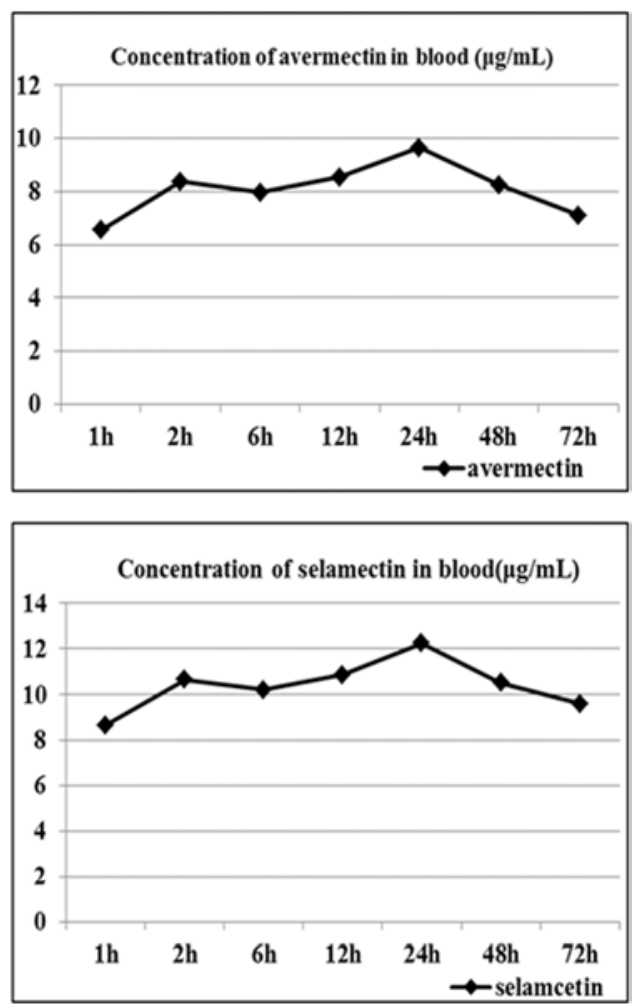

the autophagic cell death process [19-22].

Selamectin solution was widely used in pet, such as New Zealand White rabbits, bullfrogs, dogs, birds like helmeted guineafowl. Among them the dogs, cats and New Zealand Whit rabbits were in highest concentration which were respectively in $15 \mathrm{~h}, 72 \mathrm{~h}$ and $24 \mathrm{~h}$ [2, 3, 23-25]. The results are similar to the New Zealand White rabbits, in 24h. the concentration was in highest and kept a high blood concentration in longer time. The selamectin solution was also applied to exotic pet, like Golden Hamsters, helmeted guineafowl and bullfrogs $[2,3,26]$. Up to now, there were no reports on acute toxicity and adverse reactions with the addition on dosaged. So as an antiparasitic drug, it could expand its using scope in the future.

In summary, the regular concentration selamectin solution was an ideal insecticide in some parasites, like scabies mite and itch mite. There was no obviously toxic reation through the skin administration of the longissimus dorsi muscle in pets. The normal tissue structure of skin would not be damaged after administration in these sites. After given selamectin solution, the mechanism in distribution of organs 
and tissues and the skin repair induced need more further study.

\section{Conclusion}

The skin appearance changes were insignificant while the skin thickness and accessory organs increasing obviously in microscope after given more selamectin. in $24 \mathrm{~h}$, the concentration was in maximum and kept an effective blood concentration all the time. the blood and urine concentration all were in highest and kept a higher concentration until $72 \mathrm{~h}$.

\section{Funding}

This research was funded by the pharmacokinetics and skin changes in rabbit on selamectin solution (ybzysc18-31).

\section{References}

[1] M. Honda, K. Namikawa, H. Hirata, S. Neo el. (2011) An Outbreak of Trixacarus caviae Infestation in Guinea Pigs at an Animal Petting Facility and an Evaluation of the Safety and Suitable Dose of Selamectin Treatment. American Society of Parasitologists, 4 (97), 731-734.

[2] James W. Carpenter, Michael W Dryden, Butch KuKanich. (2012) Pharmacokinetics, efficacy, and adverse effects of selamectin following topical administration in flea-infested rabbits. American Journal of Veterinary Pharmacology and Therapeutics, 4 (73), 562-566.

[3] Jennifer J. Grary WestD, Dawn M. (2007) Plasma pharmacokinetics of selamectin after a single topical andministration in the american bullfrog (Rana catesbeiana). American Journal of Zoo and Wildlife Medicine, 1 (38), 51-54.

[4] Sang-Hum Kin, Jung-Youn Leet, Hyung-Kyou Jun et al. (2007) Efficacy of selamectin in the treatment of cheyletiellosis in pet rabbits. Chinese Journal of Veterinary dermatology, 19 (01), 26-27.

[5] Arif Kurtdede, Zafar Karaert, Abuzer Acar et al. (2006) Use of selamectin for the treatment of psoroptic and sarcoptic mite infestation in rabbits. Chinese Journal of Veeterinary dermatology, 18 (01), 18-22.

[6] Lara Olivieri, Giordano Nardini, Stefania Leopardi et al.(2015) Mitet infection in a masked palm civet (paguma larvata) treated by selamectin (stronghold, pfizer ltd). Journal of Zoo and Wildlife Medicine, 46 (03), 592-595.

[7] Tom L. McTier, Aleah Pullins, Sara Chapin et al.(2019) The efficacy of a novel topical formulation of selamectin plus sarolaner (Revolution Plus/Stronghold Plus) in preventing the development of Dirofilaria immitis in cats. Journal of Veterinary Parasitology, 270 (06), 56-62.

[8] Meng Yuelan. (2020) Comprehensive control of staphylococcal disease in rabbits. Chinese Journal of The Chinese Live stock and Poultry Breeding, 05 (16), 89-89.

[9] Tian Dahong. (2014) Prevention and treatment of necrotic bacillosis in rabbits. Chinese Journal of The Chinese Zhong Guo Xu Mu Shou Yi Wen Zhai, 03 (30), 105-105.
[10] Li Ming-yong, Mu Te, Liu Man etc. (2014) Isolation of Rabbit Dermatophytes and Analysis and Identification of their ITs Sequences. Chinese Journal of Rabbit Farming 06 (11), 11-14.

[11] Xu Yanhong, Zhang Yu, Yang Zhenyan. (2017) Differentiation and Treatment of Common Rabbit Skin Diseases. Chinese Journal of Rabbit Farming 01 (2017), 45-7.

[12] Wenrui Wei, Yongjun Ren, Nengxing Shen. (2019) Comparative analysis of host resistance to Sarcoptes scabiei var. cuniculi in two different rabbit breeds. Parasites \& Vectors 12 (01), 382-387.

[13] H Hernandez-Pigeon, J Besse, M Aries, S Bessou-Touya, et al. (2012) Interest of pentyl-rhamnoside in keratinocyte differentiation and cell junction for atopic dermatitis treatment [J]. Journal of Investigative Dermatology. 132: 723-748.

[14] J Idkowiak-Baldys, C Hwang, S Yim, et al. (2007) Modulation of CPT-1 expression in primary human subcutaneous adipocytes with starfruit (Averrhoa carambola) leaf extract [J]. Journal of Investigative Dermatology. 13: 151-164.

[15] M Hou, M Man, W Man, et al. (2012) Topical hesperidin improves epidermal permeability barrier function and epidermal differentiation in normal murine skin [J]. Journal of VA Med Ctr/UCSF. 21: 337-340.

[16] G Baida, P Bhalla, I Budunova. (2015) Glucocorticoids induce epidermal atrophy via activation of REDD1 and inhibition of $\mathrm{m}-\mathrm{TOR}$ activity [J]. journal of investigative dermatology. 07 : 42-85.

[17] TR Sutter, QTran, LH Kennedy, et al. (2012) EGFR regulation of epidermal barrier function [J]. Journal of physiological genomics. 03: 455-469.

[18] M Gschwandtner, M Mildner, V Mlitz, et al. (2013) Histamine suppresses epidermal keratinocyte differentiation and impairs the inside-out barrier function of the epidermis - a new role for mast cells in inflammatory skin diseases [J]. Journal of Allergy Clinical Immunology. 15: 37-47.

[19] R. C. Gupta, M. B. Masthay, T. D. Canerdy and T. M. Acosta, et al (2005) Human exposure to selamectin from dogs treated with revolution: methodological consideration for selamectin isolation [J]. Journal of Toxicology Mechanisms and Methods. 15 (04): 317-321.

[20] Vlamir Bozzatto, Patricia Rosa De Oliveira, Karim Christina Scopinho Furquim, Maria Izabel Camargo-Mathias. (2020) The occurrence of autophagic cell death in the tegument of rabbits pre-infested with rhipicephalus sanguineus and exposed to selamectin (active principle of acaricide pfizer revolution) Jourinal of Microscopy Research Technique. 76: 1171-1176.

[21] Vlamer Bozzatto, Patricia Rose de Oliveria Gervasio Henrique Bechara, et al.(2014) Morphological alterations of epidermis of rabbits infested by R. Sanguineus ticks and exposed to selamectin (active principle of pfizer revolution acaricide): A confocal microscopy study [J]. Journal of Contents lists available at Science Direct. 116: 534-538.

[22] Vlamir Bozzatto. Patricia Rose de Oliveira, Maria Izabel Camargo-Mathias.(2013) Histopathology of the tegument of rabbits infested by Rhipicephalus sanguineus (acari: ixodidae) ticks and exposed to selamectin (active principle of acaricide revolution, pfizer). Journal of Parssitol Res 24 (112), 25512560 . 
[23] R. G. Clemence a, P. Sarasola a, C. Genchi b, et al. (2000) Efficacy of selamectin in the prevention of adult heartworm (Dirofilaria immitis) infection in dogs in northern Italy. Journal of Veterinary Parasitology91: 251-258.

[24] Alicia Hahn, D. V. M, Jennifer D’Agostino, D. V. M., Dipl. A. C. Z. M., et al. (2014) Pharmacokinetics of selamectin in helmeted guineafowl (numida meleagris) after topical administration. Journal of Zoo and Wildlife Medicine 45 (1): 176-178.
[25] Sarasolap, Jerniganad, Walkerdk. (2002) Prmacokinetics of selamectin following intravenous oral and topical administration in catsanddogs. Journal of Veterinary Pharmacology and Therapeutics, 25 (4): 265-272.

[26] Hideo Hasegawa, Hiroshi Sato, Eri Iwakiri, et al. (2008) Helminths Collected From Imported Pet Murids, with Special Reference to Concomitant Infection of the Golden Hamsters with Three Pinworm Species of the Genus Syphacia (Nematoda: Oxyuridae). Journal of American Society of Parasitologists, 94 (3): 752-754. 